\title{
Optimization Scheme of Fine Toll and Bus Departure Quantity for Bottleneck Congestion Management
}

\author{
Jianhui Wu $\mathbb{D}^{1,2}$ Yuanfa Ji, ${ }^{2}$ Xiyan Sun $\mathbb{D D}^{2}$ and Yan $\mathrm{Xu}{ }^{1}$ \\ ${ }^{1}$ School of Information Science and Technology, Hunan Institute of Science and Technology, Yueyang 414006, China \\ ${ }^{2}$ Guangxi Key Laboratory of Precision Navigation Technology and Application, Guilin University of Electronic Technology, \\ Guilin 541004, China \\ Correspondence should be addressed to Xiyan Sun; sunxiyan1@163.com
}

Received 9 January 2021; Revised 3 March 2021; Accepted 8 March 2021; Published 15 March 2021

Academic Editor: Mostafa Al-Emran

Copyright (c) 2021 Jianhui Wu et al. This is an open access article distributed under the Creative Commons Attribution License, which permits unrestricted use, distribution, and reproduction in any medium, provided the original work is properly cited.

This paper chooses car travel and bus travel as the research objects, establishes a dual-mode equilibrium model based on the bottleneck model, and compares the travel characteristics of the no-toll and fine-toll schemes. We find that the fine-toll scheme can eliminate the queuing time at the bottleneck, but it also increases the congestion risk cost of bus travel. In order to eliminate the queuing time at the bottleneck and reduce the congestion risk cost of bus travel without increasing the car travel cost and bus travel cost, we propose an optimization scheme of fine toll and bus departure quantity and analyze its travel characteristics theoretically. Through the numerical example, we calculate and analyze the equilibrium results of no-toll scheme, fine-toll scheme, and optimization scheme of fine toll and bus departure quantity. The results indicate that the optimization scheme of fine toll and bus departure quantity can help travelers to choose a reasonable travel mode and travel time to travel in the rush hour.

\section{Introduction}

It is a worldwide management problem to change the phenomenon of traffic congestion in the rush hour. Especially in the central area of the city, a large number of commuting individuals gather together to form a commuting bottleneck. Vickrey [1] first proposed a bottleneck model to describe commuting behavior in the rush hour. According to the delay penalty and queuing time, travelers determine the departure time to minimize the travel cost in this model. Moreover, all individuals have the same travel cost in equilibrium. The bottleneck model can clearly reveal the formation and dissipation process of traffic congestion. On this basis, many researchers have carried out the research of commuting bottleneck management (e.g., Xiao et al. [2], Chen et al. [3], Khan and Amin [4], Guo and Sun [5], and Zhu et al. [6]). Li et al. [7] pointed out that the research on the bottleneck model in the past 50 years mainly focuses on travel behavior analysis, demand-side strategies, supply-side strategies, and joint strategies of demand and supply sides. The purpose of travel behavior analysis is to reveal the nature of congestion dynamics at the bottleneck. Relevant achievements include considerations of other travel choice dimensions (e.g., Kim [8] and Zhang et al. [9]), time-varying scheduling preferences (e.g., Abegaz et al. [10]), and vehicle physical length in queue and hypercongestion (e.g., Lamotte and Geroliminis [11]). The purpose of demand-side strategies is to reduce travel demand or redistribute the demand in space and time at the bottleneck. Relevant achievements include congestion pricing (e.g., Fosgerau and Van Dender [12]), emission pricing (e.g., Bulteau [13]), and public transit services (e.g., de Palma et al. [14]). The purpose of supplyside strategies is to determine the optimal capacity or service level of infrastructure elements. Relevant achievements include bottleneck capacity allocation (e.g., Lamotte et al. [15]) and capacity design (e.g., Qian et al. [16]). The joint strategies are the hybrid of demand-side and supply-side strategies.

From the perspective of the travel mode, the current research on commuting bottleneck management based on the bottleneck model mainly focuses on single-mode bottleneck management and multimode bottleneck management. We have summarized some research results of 
commuting bottleneck management in Table 1 . The singlemode bottleneck management based on the bottleneck model mostly regards car as the research object and guides some travelers to change their departure time by some strategies, so as to eliminate the queuing time at the commuting bottleneck. Xiao and Huang [27] pointed out that the single-step coarse toll can advance or postpone the earliest departure time and proved that their proposed piecewise time-varying toll can effectively reduce or eliminate the queues behind the bottleneck. Li et al. [28] presented the step tolling models with homogeneous and heterogeneous preferences and analyzed the optimal step toll schemes with constant and linear time-varying marginal activity utilities. Miralinaghi et al. [29] investigated the impact of a tradable credit scheme on managing morning commute congestion and proved that if commuters are equally sensitive to gain and loss, the credit allocation method does not affect the equilibrium departure rate and credit price. Wang et al. [30] proved that the total travel cost can be effectively reduced by properly allocating the capacity of high-occupancy vehicle lane and derived the optimal capacity of high-occupancy vehicle lane for minimizing the system total cost. Yu et al. [31] investigated the effect of carpooling with heterogeneous users in the bottleneck model and pointed out that if drivers choose carpooling, road capacity will be released, and this will reduce road congestion. Zhong et al. [32] believed that the high-occupancy vehicle lane can promote carpooling and the high-occupancy toll lane can bring additional welfare gains with a modest level of toll.

The multimode bottleneck management based on the bottleneck model generally takes car and public transport as the research object and guides some car travelers to take high-capacity public transport. Mirabel and Reymond [33] pointed out that the toll policy will be more efficient as long as the toll revenue is directed towards public transport when the railroad fare is equal to the average cost. Tian et al. [34] proposed a tradable credit scheme for managing bottleneck congestion and modal split and analyzed the efficiency of the tradable travel credit scheme in the highway and transit network. Li and Zhang [35] proved the critical condition for some passengers' transfer from one mode to another with congestion charging schemes and analyzed the impact of congestion charging schemes with or without internalizing the bus-related congestion externalities on the bottleneck system. At present, most of the research studies on the multimode bottleneck management based on the bottleneck model relax the interaction between car travel and public transport travel and the constraints of public transport capacity. In reality, some travelers may not be able to take the first public transport due to the difference between the limit of public transport capacity and the arrival rate of travelers, but can only wait for the next public transport. At this time, all waiting travelers need to bear the congestion risk cost of public transport.

Lin and Yang [36] assumed that the car and bus travel on the same bottleneck road and the transportation capacity of bus is limited, proposed a travel-mode equilibrium model under the condition of mixed traffic, and pointed out that the fine-toll scheme can eliminate the queuing time, but greatly increase the congestion risk cost of bus travel. Aiming at this problem, we try to guide some car travelers to choose bus travel by the fine toll and reduce the congestion risk cost of bus travel by optimizing the bus departure quantity. However, the fine toll can eliminate the queuing time of car and bus travel and also can guide some car travelers to travel by bus, but it will increase the congestion risk cost of bus travel. Increasing the bus departure quantity may extend the length of the rush hour and increase the car travel cost on the bottleneck road. Therefore, the key to the optimization scheme design of fine toll and bus departure quantity is how to ensure that it cannot only eliminate the queuing time of car and bus travel but also reduce the congestion risk cost of bus travel without increasing the travel cost of travelers.

In order to design the optimization scheme of fine toll and bus departure quantity, we take car and bus travel as the research objects and establish a dual-mode equilibrium model based on the bottleneck model in Section 2. Section 3 analyzes the travel characteristics of the no-toll and fine-toll schemes. Section 4 designs and analyzes the optimization scheme of fine-toll and bus departure quantity. In Section 5, a numerical example is presented to validate the theoretical results. Finally, conclusions are given in Section 6 .

\section{Dual-Mode Equilibrium Model}

To analyze the influence of fine toll and bus departure quantity on travelers' travel mode choice at the bottleneck, we suppose that there is a bottleneck road with limited capacity at the entrance of living area $O$ to work area $D$, the maximum capacity of the bottleneck road is $s$, the bus departure quantity on the bottleneck road is $f_{0}$, the maximum passenger capacity of the unit bus is $d$, and travelers are completely rational, have complete traffic information, and can only choose to travel by car or bus on the bottleneck road. Suppose that the travel time for an individual who leaves living area $O$ at time $t$ is $T(t)$, the free-flow travel time from living area $O$ to work area $D$ is $T_{f}$, the queuing time from living area $O$ to work area $D$ at time $t$ is $T_{w}(t)$, $T(t)=T_{f}+T_{w}(t)$, the departure time at which an individual arrives at work area $D$ at time $t^{*}$ is $t_{0}$, $t_{0}+T_{f}+T_{w}\left(t_{0}\right)=t^{*}$, the car departure time at which the queue begins is $t_{1 e}$, the car departure time at which the queue ends is $t_{1 l}$, the bus departure time at which the queue begins is $t_{2 e}$, the bus departure time at which the queue ends is $t_{2 l}$, the congestion risk cost of bus travel at time $t$ is $R(t)$, the bus ticket price is $p$, the unit cost of car travel time is $\alpha_{1}$, the unit cost of bus travel time is $\alpha_{2}$, the unit cost of an early arrival is $\beta$, the unit cost of a late arrival is $\gamma, \gamma>\alpha_{1}>\alpha_{2}>\beta, \phi$ and $\theta$ are the weight coefficient, the number of travelers departing from living area $O$ to work area $D$ every morning is $N$, the number of travelers who choose to go to work area $D$ by car is $N_{1}$, the number of travelers who choose to go to work area $D$ by bus is $N_{2}$, and all travelers expect to arrive at work area $D$ at time $t^{*}$.

Considering that the fuel cost is related to travel time, we convert it into the travel time $T$. Based on this, we suppose that the car travel cost $C_{1}$ is the weighted sum of free-flow 
TABLE 1: Some research results of commuting bottleneck management.

\begin{tabular}{lccc}
\hline Literatures & $\begin{array}{c}\text { Type of travel } \\
\text { mode }\end{array}$ & Decision variable & Target \\
\hline Ge et al. [17] & Single mode & Toll & Minimum generalized cost \\
Lindsey et al. [18] & Single mode & Step toll & Toll levels and tolling periods optimal \\
Laih [19] & Sliminate queuing time \\
Miralinaghi and Peeta & Single mode & Tradable credit & Minimize emissions \\
[20] & Single mode & Ridesharing payment and & parking charge \\
Ma and Zhang [21] & Single mode & Toll & Eliminate queuing time \\
Liu and Li [22] & Multimode & Tradable parking permit & System optimal \\
Xiao et al. [23] & Multimode & Vehicle lane & System optimal \\
Chen et al. [24] & Multimode & Tradable credit & Minimize social cost \\
Seilabi et al. [25] & Multimode & Toll & Minimize total travel time \\
Holguín-Veras and & Multimode & Fine toll and bus departure & Eliminate queuing time and reduce congestion risk cost \\
Cetin [26] & quantity & without increasing travel cost \\
This paper & & &
\end{tabular}

travel time, queuing time, and delay time of early or late arrival. This can be expressed as

$$
C_{1}(t)= \begin{cases}\alpha_{1} T(t)+\beta\left(t^{*}-T(t)-t\right), & \forall t \in\left[t_{1 e}, t_{0}\right], \\ \alpha_{1} T(t)+\gamma\left(t-t^{*}+T(t)\right), & \forall t \in\left(t_{0}, t_{1 l}\right] .\end{cases}
$$

We define the bus travel cost $C_{2}$ as the weighted sum of walking time, waiting time, free-flow travel time, queuing time, delay time of early or late arrival, congestion risk cost, bus ticket price, and discomfort cost caused by physical contact. Assume that the walking time is constant, we convert it into the bus ticket price $p$. The waiting time is only the time to wait for the first bus to arrive. The waiting time for the next bus caused by the congestion in the first bus is represented by the congestion risk cost. As the bus timetable is known, travelers will reduce their waiting time as much as possible. For this reason, we assume that the waiting time is equal to zero. Meanwhile, if travelers choose to travel in the rush hour, they have fully considered that the bus is crowded with a large number of passengers. For this reason, we assume that the discomfort cost caused by physical contact is constant and convert it into the bus ticket price $p$. Based on these assumptions, the bus travel cost $C_{2}$ can be expressed as

$$
C_{2}(t)= \begin{cases}\alpha_{2} T(t)+\beta\left(t^{*}-T(t)-t\right)+\phi R(t)+p, & \forall t \in\left[t_{2 e}, t_{0}\right], \\ \alpha_{2} T(t)+\gamma\left(t-t^{*}+T(t)\right)+\theta R(t)+p, & \forall t \in\left(t_{0} t_{2 l}\right] .\end{cases}
$$

In order to reduce individual travel cost, travelers choose departure time by weighing travel time and delay cost. For car travelers, there are not queues at time $t_{1 e}$ and $t_{1 l}$, and the queuing time $T_{w}\left(t_{1 e}\right)=T_{w}\left(t_{1 l}\right)=0$. When no traveler can reduce the travel cost by changing the departure time, the traffic flow on the road reaches equilibrium. At equilibrium, all travelers have the same travel cost. It can be expressed as

$$
\begin{aligned}
C_{1}\left(t_{1 e}\right) & =C_{1}\left(t_{1 l}\right)=\alpha_{1} T_{f}+\beta\left(t^{*}-T_{f}-t_{1 e}\right) \\
& =\alpha_{1} T_{f}+\gamma\left(t_{1 l}-t^{*}+T_{f}\right) .
\end{aligned}
$$

Since cars and buses run on the same route, the length of the rush hour is affected by both individual car travelers and individual bus travelers. Hence, the length of the rush hour on the car travel route can be expressed as

$$
t_{1 l}-t_{1 e}=\frac{N_{1}+\lambda\left(t_{1 l}-t_{1 e}\right) f_{0}}{s} .
$$

Evidently,

$$
t_{1 l}-t_{1 e}=\frac{N_{1}}{s-\lambda f_{0}}
$$

where $\lambda$ represents the conversion coefficient between a bus and an equivalent car.

When the car travel reaches equilibrium, we can obtain the car travel cost $C_{1}$, the car departure time $t_{1 e}$ at which the queue begins, and the car departure time $t_{1 l}$ at which the queue ends by formulas (1), (3), and (5). It can be expressed as

$$
\begin{aligned}
& C_{1}=\alpha_{1} T_{f}+\frac{\beta \gamma}{\beta+\gamma} \frac{N_{1}}{s-\lambda f_{0}}, \\
& t_{1 e}=t^{*}-T_{f}-\frac{\gamma}{\beta+\gamma} \frac{N_{1}}{s-\lambda f_{0}}, \\
& t_{1 l}=t^{*}-T_{f}+\frac{\beta}{\beta+\gamma} \frac{N_{1}}{s-\lambda f_{0}}, \\
& t_{0}=t^{*}-T_{f}-\frac{\beta \gamma}{\alpha_{1}(\beta+\gamma)} \frac{N_{1}}{s-\lambda f_{0}} .
\end{aligned}
$$

For bus travelers at time $t_{2 e}$ and $t_{2 l}$, they do not queue up, their queuing time $T_{w}\left(t_{2 e}\right)=T_{w}\left(t_{2 l}\right)=0$, and the congestion risk cost $R\left(t_{2 e}\right)=R\left(t_{2 l}\right)=0$, but the delay cost of early or late arrival is higher. For bus travelers between time $t_{2 e}$ and $t_{2 l}$, their delay cost of early or late arrival is decreased, but their queuing time and congestion risk cost are increased as congestion. When the equilibrium is reached, the bus travel cost is equal at all times. It can be expressed as 


$$
\begin{aligned}
C_{2}\left(t_{2 e}\right) & =C_{2}\left(t_{2 l}\right)=\alpha_{2} T_{f}+\beta\left(t^{*}-T_{f}-t_{2 e}\right)+p \\
& =\alpha_{2} T_{f}+\gamma\left(t_{2 l}-t^{*}+T_{f}\right)+p .
\end{aligned}
$$

Since the bus passenger capacity is limited, we have

$$
t_{2 e}-t_{2 l}=\frac{N_{2}}{f_{0} d} .
$$

When the bus travel reaches equilibrium, we can obtain the bus travel $\operatorname{cost} C_{2}$, the bus departure time $t_{2 e}$ at which the queue begins, and the bus departure time $t_{2 l}$ at which the rush hour queue ends by formulas (10) and (11). It can be expressed as

$$
\begin{aligned}
& C_{2}=\alpha_{2} T_{f}+p+\frac{\beta \gamma}{\beta+\gamma} \frac{N_{2}}{f_{0} d}, \\
& t_{2 e}=t^{*}-T_{f}-\frac{\gamma}{\beta+\gamma} \frac{N_{2}}{f_{0} d}, \\
& t_{2 l}=t^{*}-T_{f}+\frac{\beta}{\beta+\gamma} \frac{N_{2}}{f_{0} d} .
\end{aligned}
$$

\section{Travel Characteristics under No Toll and Fine Toll}

3.1. Travel Characteristics under No Toll. If there is no one traveler who can change his travel mode or departure time to reduce travel cost, the road traffic flow reaches the equilibrium state. At this time, the car travel cost is equal to the bus travel cost, that is, $C_{1}=C_{2}$. Hence, we can obtain the travel distribution of the car and bus by formulas (6) and (12) at equilibrium. It can be expressed as

$$
\left\{\begin{array}{l}
N_{1}=\frac{s-\lambda f_{0}}{s+(d-\lambda) f_{0}}\left[N-\frac{f_{0} d(\beta+\gamma)}{\beta \gamma}\left(\alpha_{1} T_{f}-\alpha_{2} T_{f}-p\right)\right] \\
N_{2}=N-N_{1} .
\end{array}\right.
$$

The system total travel cost $S C$ can be expressed as

$$
\mathrm{SC}=\left(\alpha_{1} T_{f}+\frac{\beta \gamma}{\beta+\gamma} \frac{N_{1}}{s-\lambda f_{0}}\right) N .
$$

Since cars and buses share the same route at the bottleneck, they have to bear the same queuing time when they start at the same time. According to formulas (1), (6), and (7), the queuing time $T_{w}(t)$ can be written as

$$
T_{w}(t)= \begin{cases}0, & \forall t \in\left[t_{2 e}, t_{1 e}\right], \\ \frac{\beta}{\alpha_{1}-\beta}\left(t-t_{1 e}\right), & \forall t \in\left(t_{1 e}, t_{0}\right], \\ \frac{\gamma}{\alpha_{1}+\gamma}\left(t_{1 l}-t\right), & \forall t \in\left(t_{0}, t_{1 l}\right], \\ 0, & \forall t \in\left(t_{1 l}, t_{2 l}\right] .\end{cases}
$$

Using formulas (2), (12)-(14), and (17), the congestion risk cost $R(t)$ of bus travel can be expressed as

$$
R(t)= \begin{cases}\frac{\beta}{\phi}\left(t-t_{2 e}\right), & \forall t \in\left[t_{2 e}, t_{1 e}\right], \\ \frac{\beta\left(\alpha_{1}-\alpha_{2}\right)}{\phi\left(\alpha_{1}-\beta\right)} t-\frac{\beta}{\phi} t_{2 e}+\frac{\beta\left(\alpha_{2}-\beta\right)}{\phi\left(\alpha_{1}-\beta\right)} t_{1 e}, & \forall t \in\left(t_{1 e}, t_{0}\right], \\ \frac{\gamma}{\theta} t_{2 l}-\frac{\gamma\left(\alpha_{2}+\gamma\right)}{\theta\left(\alpha_{1}+\gamma\right)} t_{1 l}-\frac{\gamma\left(\alpha_{1}-\alpha_{2}\right)}{\theta\left(\alpha_{1}+\gamma\right)} t, & \forall t \in\left(t_{0}, t_{1 l}\right], \\ \frac{\gamma}{\theta}\left(t_{2 l}-t\right), & \forall t \in\left(t_{1 l}, t_{2 l}\right) .\end{cases}
$$

3.2. Travel Characteristics under Fine Toll. The fine-toll scheme is a dynamic charging strategy, which is actually collecting the road usage toll converted by queuing time from car travelers and achieving the social optimal state by changing the time distribution of car travel. The fine toll $\pi$ can be written as follows (Arnott et al. [37]):

$$
\pi(t)= \begin{cases}\frac{\beta \gamma}{\beta+\gamma} \frac{N_{1}}{s-\lambda f_{0}}-\beta\left(t^{*}-T_{f}-t\right), & \forall t \in\left[t_{1 e}, t^{*}-T_{f}\right], \\ \frac{\beta \gamma}{\beta+\gamma} \frac{N_{1}}{s-\lambda f_{0}}-\gamma\left(t-t^{*}+T_{f}\right), & \forall t \in\left(t^{*}-T_{f}, t_{1 l}\right] .\end{cases}
$$

Since the fine-toll scheme does not increase the car travel cost, the demand distribution of car travel and bus travel under the fine-toll scheme is the same as that of the no-toll scheme. In addition, the system total travel cost SC under the fine-toll scheme can be written as

$$
\mathrm{SC}=\left(\alpha_{1} T_{f}+\frac{\beta \gamma}{\beta+\gamma} \frac{N_{1}}{s-\lambda f_{0}}\right) N .
$$

The fine-toll scheme eliminates the queuing time of car and bus travel on the road $\left(T_{w}(t)=0\right)$, but the congestion risk cost of bus travel is different from that of the no-toll scheme. According to formulas (2) and (12)-(14), the congestion risk cost $R(t)$ of bus travel can be expressed as 


$$
R(t)= \begin{cases}\frac{\beta}{\phi}\left(t-t_{2 e}\right), & \forall t \in\left[t_{2 e}, t^{*}-T_{f}\right], \\ \frac{\gamma}{\theta}\left(t_{2 l}-t\right), & \forall t \in\left(t^{*}-T_{f}, t_{2 l}\right] .\end{cases}
$$

\section{Optimization Scheme of Fine Toll and Bus Departure Quantity}

Comparing formulas (18) and (21), it is not difficult to find that the fine-toll scheme increases the congestion risk cost of bus travel from $t_{1 e}$ to $t_{1 l}$. To overcome this problem, this section attempts to find an optimization scheme of fine toll and bus departure quantity, which cannot only eliminate the queuing time of car and bus travel on the road but also reduce the congestion risk cost of bus travel without increasing the travel cost of travelers.

4.1. Scheme Formulation. In the whole rush hour, the bottleneck road is operating at full capacity; then, the total cost $T C_{1}$ of car travel can be expressed as

$$
T C_{1}=\int_{t_{1 e}}^{t_{1 l}}\left(s-\lambda f_{0}\right) C_{1}(t) \mathrm{d} t
$$

By substituting formula (1) into formula (22), we can obtain the total cost $T C_{1}$ of car travel under the no-toll scheme when the queuing time $T_{w}(t)=0$. It can be written as

$$
\begin{aligned}
T C_{1}= & \int_{t_{1 e}}^{t^{*}-T_{f}}\left(s-\lambda f_{0}\right)\left[\alpha_{1} T_{f}+\beta\left(t^{*}-T_{f}-t\right)\right] \mathrm{d} t \\
& +\int_{t^{*}-T_{f}}^{t_{1 l}}\left(s-\lambda f_{0}\right)\left[\alpha_{1} T_{f}+\gamma\left(t-t^{*}+T_{f}\right)\right] \mathrm{d} t \\
= & \alpha_{1} T_{f} N_{1}+\frac{\beta \gamma}{2(\beta+\gamma)} \frac{N_{1}^{2}}{s-\lambda f_{0}} .
\end{aligned}
$$

According to formula (23), we can obtain the average car travel cost $\bar{C}_{1}$ under the no-toll scheme when the queuing time $T_{w}(t)=0$. It can be expressed as

$$
\bar{C}_{1}=\frac{T C_{1}}{N_{1}}=\alpha_{1} T_{f}+\frac{\beta \gamma}{2(\beta+\gamma)} \frac{N_{1}}{s-\lambda f_{0}} .
$$

Suppose that the bus departure quantity is $f_{0}\left(f_{b}>f_{0}\right)$ and the fine toll is $\pi^{\prime}(t)$ under the optimization scheme of fine toll and bus departure quantity. Then, formula (1) can be rewritten as

$$
C_{1}^{\prime}(t)= \begin{cases}\alpha_{1} T_{f}+\beta\left(t^{*}-T_{f}-t\right)+\pi^{\prime}(t), & \forall t \in\left[t_{1 e}^{\prime}, t^{*}-T_{f}\right], \\ \alpha_{1} T_{f}+\gamma\left(t-t^{*}+T_{f}\right)+\pi^{\prime}(t), & \forall t \in\left(t^{*}-T_{f}, t_{1 l}^{\prime}\right] .\end{cases}
$$

For car travelers at time $t_{1 e}{ }^{\prime}$ and $t_{1 l}^{\prime}$, they do not have to pay a fine toll, that is, $\pi^{\prime}\left(t_{1 l}^{\prime}\right)=\pi^{\prime}\left(t_{1 l}^{\prime}\right)=0$. Referring to the solution steps under the no-toll scheme, it is not difficult to get the car travel cost $C_{1}^{\prime}$ and the bus travel cost $C_{2}^{\prime}$ at equilibrium. It can be expressed as

$$
\begin{aligned}
& C_{1}^{\prime}=\alpha_{1} T_{f}+\frac{\beta \gamma}{\beta+\gamma} \frac{N_{1}^{\prime}}{s-\lambda f_{b}}, \\
& C_{2}^{\prime}=\alpha_{2} T_{f}+p+\frac{\beta \gamma}{\beta+\gamma} \frac{N_{2}^{\prime}}{f_{b} d},
\end{aligned}
$$

where $N_{1}^{\prime}$ represents the number of travelers who choose to go to work area $D$ by car and $N_{2}^{\prime}$ is the number of travelers who choose to go to work area $D$ by bus. According to formulas (26) and (27), we have

$$
\left\{\begin{array}{l}
N_{1}^{\prime}=\frac{s-\lambda f_{b}}{s+(d-\lambda) f_{b}}\left[N-\frac{f_{b} d(\beta+\gamma)}{\beta \gamma}\left(\alpha_{1} T_{f}-\alpha_{2} T_{f}-p\right)\right], \\
N_{2}^{\prime}=N-N_{1}^{\prime} .
\end{array}\right.
$$

In order to ensure that the optimization scheme of fine toll and bus departure quantity does not increase the travel cost of travelers, we suppose that $C_{1}^{\prime}=\bar{C}_{1}$. According to formulas (24) and (26), we know

$$
\frac{1}{2} \frac{N_{1}}{s-\lambda f_{0}}=\frac{N_{1}^{\prime}}{s-\lambda f_{b}} .
$$

According to formulas (15), (28), and (29), we have

$$
f_{b}=\frac{\left[s+2 f_{0}(d-\lambda)\right] \beta \gamma N+s f_{0} d(\beta+\gamma)\left(\alpha_{1} T_{f}-\alpha_{2} T_{f}-p\right)}{(d-\lambda) \beta \gamma N+d\left[2 s+f_{0}(d-\lambda)\right](\beta+\gamma)\left(\alpha_{1} T_{f}-\alpha_{2} T_{f}-p\right)} .
$$

According to formulas (25), (26), and (29), we have

$$
\pi^{\prime}(t)= \begin{cases}\frac{\beta \gamma}{2(\beta+\gamma)} \frac{N_{1}}{s-\lambda f_{0}}-\beta\left(t^{*}-T_{f}-t\right), & \forall t \in\left[t_{1 e}{ }^{\prime}, t^{*}-T_{f}\right], \\ \frac{\beta \gamma}{2(\beta+\gamma)} \frac{N_{1}}{s-\lambda f_{0}}-\gamma\left(t-t^{*}+T_{f}\right), & \forall t \in\left(t^{*}-T_{f}, t_{1 l}^{\prime}\right] .\end{cases}
$$

4.2. Travel Characteristics' Analysis. From the scheme formulation in the previous section, we can know that the optimization scheme of fine toll and bus departure quantity eliminates the queuing time of car and bus travel on the road and also changes the demand distribution of car and bus travel. Next, we will analyze these travel characteristics of this proposed scheme including the length of the rush hour, congestion risk cost, and system total travel cost.

4.2.1. The Length of the Rush Hour. Referring to the solution steps of the car departure time at which the queue begins and ends under the no-toll scheme, it is not difficult to get the car departure time $t_{1 e}^{\prime}$ at which the queue begins, the car departure time $t_{1 l}^{\prime}$ at which the queue ends, the bus departure 
time $t_{2 e}^{\prime}$ at which the queue begins, and the bus departure time $t_{2 l}^{\prime}$ at which the queue ends. It can be expressed as

$$
\begin{aligned}
& t_{1 e}^{\prime}=t^{*}-T_{f}-\frac{\gamma}{\beta+\gamma} \frac{N_{1}^{\prime}}{s-\lambda f_{b}}, \\
& t_{1 l}^{\prime}=t^{*}-T_{f}+\frac{\beta}{\beta+\gamma} \frac{N_{1}^{\prime}}{s-\lambda f_{b}}, \\
& t_{2 e}^{\prime}=t^{*}-T_{f}-\frac{\gamma}{\beta+\gamma} \frac{N_{2}^{\prime}}{f_{b} d}, \\
& t_{2 l}^{\prime}=t^{*}-T_{f}+\frac{\beta}{\beta+\gamma} \frac{N_{2}^{\prime}}{f_{b} d} .
\end{aligned}
$$

According to formula (28), we can know that $N_{1}^{\prime}<N_{1}$. In addition, since $f_{b}>f_{0}$, then $N_{1}^{\prime} / s-\lambda f_{b}<N_{1} / s-\lambda f_{0}$. Comparing formulas (7), (8), and (32), we can know that $t_{1 e}^{\prime}>t_{1 e}$ and $t_{1 l}^{\prime}<t_{1 l}$, that is, $t_{1 l}^{\prime}-t_{1 e}^{\prime}<t_{1 l}-t_{1 e}$.

Moreover, according to formulas (6), (12), (26), (27), and (29), we have

$$
\begin{gathered}
\alpha_{1} T_{f}+\frac{\beta \gamma}{\beta+\gamma} \frac{N_{1}}{s-\lambda f_{0}}=\alpha_{2} T_{f}+p+\frac{\beta \gamma}{\beta+\gamma} \frac{N_{2}}{f_{0} d}, \\
\alpha_{1} T_{f}+\frac{\beta \gamma}{2(\beta+\gamma)} \frac{N_{1}}{s-\lambda f_{0}}=\alpha_{2} T_{f}+p+\frac{\beta \gamma}{\beta+\gamma} \frac{N_{2}^{\prime}}{f_{b} d} .
\end{gathered}
$$

By substracting formula (35) from formula (34), we have

$$
\frac{N_{2}^{\prime}}{f_{b} d}<\frac{N_{2}}{f_{0} d} .
$$

Comparing formulas (13), (14), and (33), we can know that $t_{2 e}^{\prime}>t_{2 e}$ and $t_{2 l}^{\prime}<t_{2 l}$, that is, $t_{2 l}^{\prime}-t_{2 e}^{\prime}<t_{2 l}-t_{2 e}$.

To sum up, it can be seen that the optimization scheme of fine toll and bus departure quantity reduces the length of the rush hour on the bottleneck road. This shows that the optimization scheme of fine toll and bus departure quantity can change the travel time distribution of car travelers and bus travelers.

4.2.2. Congestion Risk Cost. Referring to the solution steps of the congestion risk cost under the no-toll scheme, the congestion risk cost $R^{\prime}$ of bus travel can be expressed as

$$
R^{\prime}(t)= \begin{cases}\frac{\beta}{\phi}\left(t-t_{2 e}^{\prime}\right), & \forall t \in\left[t_{2 e}^{\prime}, t^{*}-T_{f}\right], \\ \frac{\gamma}{\theta}\left(t_{2 l}^{\prime}-t\right), & \forall t \in\left(t^{*}-T_{f}, t_{2 l}^{\prime}\right] .\end{cases}
$$

Comparing formulas (21) and (37), we can know that the congestion risk cost of bus travel under the optimization scheme of fine toll and bus departure quantity is smaller than that of the fine-toll scheme. This also reflects that appropriately increasing the bus departure quantity in the rush hour can effectively reduce the congestion risk cost of bus travel.
4.2.3. System Total Travel Cost. According to formulas (26) and (29), the system total travel cost $S C^{\prime}$ under the optimization scheme of fine toll and bus departure quantity can be written as

$$
S C^{\prime}=\left(\alpha_{1} T_{f}+\frac{\beta \gamma}{2(\beta+\gamma)} \frac{N_{1}}{s-\lambda f_{0}}\right) N .
$$

Comparing formulas (16), (20), and (38), we can find that the system total travel cost under the optimization scheme of fine toll and bus departure quantity is smaller than that of the fine-toll scheme and the no-toll scheme. This also reflects that the optimization scheme of fine toll and bus departure quantity can help travelers to choose a reasonable travel mode and travel time to travel in the rush hour.

\section{Numerical Experiments}

To verify the validity of the proposed optimization scheme, we suppose that there is a bottleneck road with limited capacity at the entrance of living area $O$ to work area $D$, the maximum bottleneck capacity $s=2000$ vehicle/hour, 6000 $(N=6000)$ travelers departing from living area $O$ to work area $D$ every morning, and all travelers expect to arrive at work area $D$ at 8 a.m. $\left(t^{*}=8\right)$. Meanwhile, we suppose that the free-flow travel time $T_{f}=0.5$ hour from living area $O$ to work area $D$, the maximum passenger capacity $d=40$ of the unit bus, the bus departure quantity $f_{0}=50$, the bus ticket price $p=1$, and the system parameter $\lambda=2, \alpha_{1}=7, \alpha_{2}=4$, $\beta=0.6, \gamma=9, \phi=1$, and $\theta=1$, and the fine toll at the bottleneck road is realized by the electronic toll collection system.

Next, we will calculate and analyze these schemes including the no-toll scheme (NT), fine-toll scheme (FT), and optimization scheme of fine toll and bus departure quantity (FT-BDQ). For the ease of comparison, some formulas for NT, FT, and FT-BDQ schemes are listed in Table 2. The results of calculation are as follows.

In Table 3, we can observe that travel cost and system total travel cost under the optimization scheme of fine toll and bus departure quantity are smaller than that of the finetoll scheme and the no-toll scheme. This reflects that the optimization scheme of fine toll and bus departure quantity can reduce travelers' travel cost. Comparing the equilibrium results of the 2nd column and 3rd column in Table 3, we can know that appropriately increasing the bus departure quantity in the rush hour can attract some travelers to travel by bus. This means that the optimization scheme of fine toll and bus departure quantity can effectively guide travelers to choose a reasonable travel mode to travel in the rush hour.

In Figure 1, we can observe that the congestion risk cost of bus travel under the optimization scheme of fine toll and bus departure quantity may be higher than that of the no-toll scheme in some times of the rush hour, but it is lower than that of the fine-toll scheme in the rush hour. This also reflects that appropriately increasing the bus departure quantity on the basis of the fine toll can effectively reduce the congestion risk cost of bus travel. 


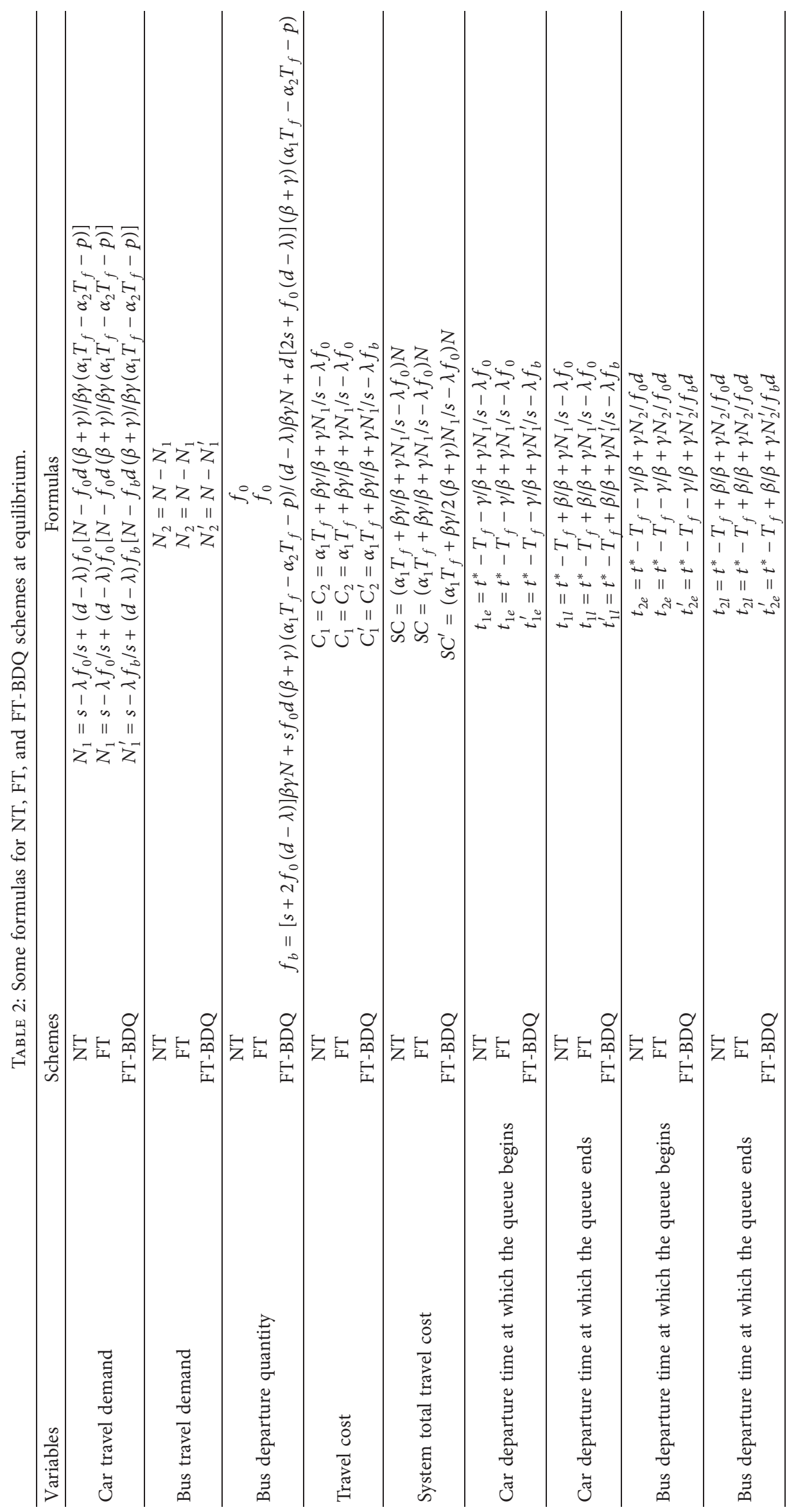


TABLE 3: Equilibrium results under three schemes.

\begin{tabular}{lccccc}
\hline Schemes & Car travel demand & Bus travel demand & Bus departure quantity & Travel cost & System total travel cost \\
\hline NT & 2056.9800 & 3943.0199 & 50 & 4.1090 & 24653.8461 \\
FT & 2056.9800 & 3943.0199 & 50 & 4.1090 & 24653.8461 \\
FT-BDQ & 987.7681 & 5012.2319 & 87.6142 & 3.8045 & 22826.9230 \\
\hline
\end{tabular}

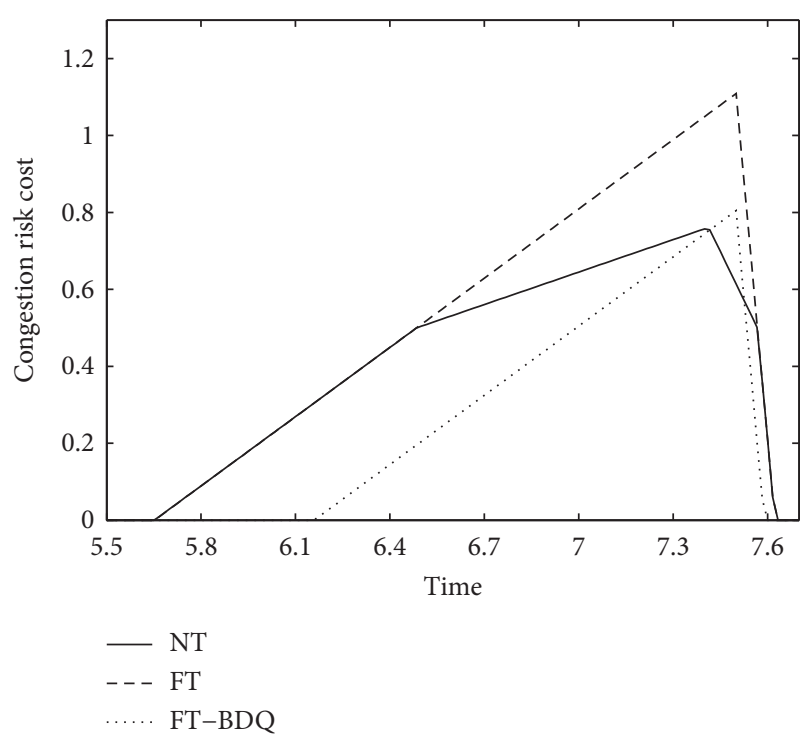

Figure 1: Congestion risk cost of bus travel under three schemes.

TABLE 4: Travel time distribution of car travelers and bus travelers under three schemes.

\begin{tabular}{lcccc}
\hline Schemes & $t_{1 e}\left(\right.$ or $\left.t_{1 e}^{\prime}\right)$ & $t_{2 e}\left(\right.$ or $\left.t_{2 e}^{\prime}\right)$ & $t_{1 l}\left(\right.$ or $\left.t_{1 l}^{\prime}\right)$ & $t_{2 l}\left(\right.$ or $\left.t_{2 l}^{\prime}\right)$ \\
\hline NT & $6: 29$ & $5: 39$ & $7: 34$ & $7: 37$ \\
FT & $6: 29$ & $5: 39$ & $7: 34$ & $7: 37$ \\
FT-BDQ & $6: 59$ & $6: 09$ & $7: 32$ & $7: 35$ \\
\hline
\end{tabular}

In Table 4, we can observe that the car and bus departure time at which the queue begins under the optimization scheme of fine toll and bus departure quantity are later than that of the fine-toll scheme and the no-toll scheme; the car and bus departure time at which the queue ends under the optimization scheme of fine toll and bus departure quantity are earlier than that of the fine-toll scheme and the no-toll scheme. This also shows that the optimization scheme of fine toll and bus departure quantity cannot only help travelers to choose a reasonable travel time to travel in the rush hour but also shorten the rush hour of car and bus travel.

\section{Conclusions}

In this paper, we established a dual-mode equilibrium model based on the bottleneck model. In order to overcome the problem that the fine-toll scheme increases the congestion risk cost of bus travel, we proposed an optimization scheme of fine toll and bus departure quantity and demonstrated its travel characteristics from the perspective of theoretical analysis and numerical simulation. The findings from these experiments are summarized as follows:
(1) The optimization scheme of fine toll and bus departure quantity can reduce the system total travel cost and travelers' travel cost and help travelers to choose a reasonable travel mode to travel in the rush hour.

(2) The congestion risk cost of bus travel under the optimization scheme of fine toll and bus departure quantity is lower than that of the fine-toll scheme in the rush hour. Appropriately increasing the bus departure quantity can effectively reduce the congestion risk cost of bus travel.

(3) The optimization scheme of fine toll and bus departure quantity cannot only guide travelers to choose a reasonable travel time to travel in the rush hour but also shorten the rush hour of car and bus travel.

The bottleneck of road and public transportation is the key point to traffic congestion. Therefore, this study can help alleviate the urban traffic congestion and promote the development of urban traffic demand management methods.

With regard to the above finding, it is worth further looking at how to find the "best" simple scheme of fine toll and bus departure quantity under uncertain demand. Another interesting direction for further research is how to consider the influence of travel-mode choice behavior heterogeneity on the optimization scheme of fine toll and bus departure quantity. Additionally, the measurement of the congestion risk cost is also our future research direction.

\section{Data Availability}

The data used to support the findings of this study are available from the corresponding author upon request.

\section{Conflicts of Interest}

The authors declare that there are no conflicts of interest regarding the publication of this paper.

\section{Acknowledgments}

This research work was supported by the National Natural Science Foundation of China (Grant nos. 61561016 and 61861008), National Key R\&D Program of China (Grant no. 2018YFB0505103), Hunan Provincial Natural Science Foundation of China (Grant no. 2019JJ50210), Scientific Research Foundation of Hunan Provincial Education Department (Grant no. 19B234), Foundation from the Guangxi Zhuang Autonomous Region (Grant nos. AC16380014, AA17202048, and AA17202033), and Guangxi Key 
Laboratory of Precision Navigation Technology and Application (Grant no. DH202009).

\section{References}

[1] W. Vickery, "Congestion theory and transport investment," American Economic Review, vol. 59, no. 2, pp. 251-260, 1969.

[2] L. Xiao, H. Huang, and L. Tian, "Stochastic bottleneck model with heterogeneous travelers," Journal of Transportation Systems Engineering and Information Technology, vol. 14, no. 4, pp. 93-98, 2014.

[3] D. Chen, N. Zhang, and L. J. Liu, "Bottleneck model for biarrival time: principle of staggered work hours," Journal of Management Sciences in China, vol. 18, no. 8, pp. 52-60, 2015.

[4] Z. Khan and S. Amin, "Bottleneck model with heterogeneous information," Transportation Research Part B: Methodological, vol. 112, pp. 157-190, 2018.

[5] X. Guo and H. J. Sun, "Modeling the morning commute problem in a bottleneck model based on personal perception," Journal of Advanced Transportation, vol. 2019, Article ID 6184827, 12 pages, 2019.

[6] Z. Zhu, X. Li, W. Liu, and H. Yang, "Day-to-day evolution of departure time choice in stochastic capacity bottleneck models with bounded rationality and various information perceptions," Transportation Research Part E: Logistics and Transportation Review, vol. 131, pp. 168-192, 2019.

[7] Z.-C. Li, H.-J. Huang, and H. Yang, "Fifty years of the bottleneck model: a bibliometric review and future research directions," Transportation Research Part B: Methodological, vol. 139, pp. 311-342, 2020.

[8] J. Kim, "Estimating the social cost of congestion using the bottleneck model," Economics of Transportation, vol. 19, pp. 1-19, 2019.

[9] X. Zhang, W. Liu, S. T. Waller, and Y. Yin, "Modelling and managing the integrated morning-evening commuting and parking patterns under the fully autonomous vehicle environment," Transportation Research Part B: Methodological, vol. 128, pp. 380-407, 2019.

[10] D. Abegaz, K. Hjorth, and J. Rich, "Testing the slope model of scheduling preferences on stated preference data," Transportation Research Part B: Methodological, vol. 128, pp. 380407, 2019.

[11] R. Lamotte and N. Geroliminis, "The morning commute in urban areas with heterogeneous trip lengths," Transportation Research Part B: Methodological, vol. 117, pp. 794-810, 2018.

[12] M. Fosgerau and K. Van Dender, "Road pricing with complications," Transportation, vol. 40, no. 3, pp. 479-503, 2013.

[13] J. Bulteau, "Revisiting the bottleneck congestion model by considering environmental costs and a modal policy," International Journal of Sustainable Transportation, vol. 10, no. 3, pp. 180-192, 2016.

[14] A. de Palma, R. Lindsey, and G. Monchambert, "The economics of crowding in rail transit," Journal of Urban Economics, vol. 101, pp. 106-122, 2017.

[15] R. Lamotte, A. de Palma, and N. Geroliminis, "On the use of reservation-based autonomous vehicles for demand management," Transportation Research Part B: Methodological, vol. 99, pp. 205-227, 2017.

[16] Z. Qian, F. Xiao, and H. M. Zhang, "Managing morning commute traffic with parking," Transportation Research Part B: Methodological, vol. 46, no. 7, pp. 894-916, 2012.

[17] Y. E. Ge, K. Stewart, Y. D. Liu, C. Y. Tang, and B. Z. Liu, "Investigating boundary effects of congestion charging in a single bottleneck scenario," Transport, vol. 33, no. 1, pp. 7791, 2018.

[18] C. R. Lindsey, V. A. C. V. D. Berg, and E. T. Verhoef, "Step tolling with bottleneck queuing congestion," Journal of Urban Economics, vol. 72, no. 1, pp. 46-59, 2012.

[19] C.-H. Laih, "Queueing at a bottleneck with single- and multistep tolls," Transportation Research Part A: Policy and Practice, vol. 28, no. 3, pp. 197-208, 1994.

[20] M. Miralinaghi and S. Peeta, "Promoting zero-emissions vehicles using robust multi-period tradable credit scheme," Transportation Research Part D: Transport and Environment, vol. 75, pp. 265-285, 2019.

[21] R. Ma and H. M. Zhang, "The morning commute problem with ridesharing and dynamic parking charges," Transportation Research Part B: Methodological, vol. 106, pp. 345374, 2017.

[22] Y. Liu and Y. Li, "Pricing scheme design of ridesharing program in morning commute problem," Transportation Research Part C: Emerging Technologies, vol. 79, pp. 156-177, 2017.

[23] L. L. Xiao, T. L. Liu, and H. J. Huang, "Tradable permit schemes for managing morning commute with carpool under parking space constraint," Transportation, vol. 1, pp. 1-24, 2019.

[24] Z. Chen, F. He, L. Zhang, and Y. Yin, "Optimal deployment of autonomous vehicle lanes with endogenous market penetration," Transportation Research Part C: Emerging Technologies, vol. 72, pp. 143-156, 2016.

[25] S. E. Seilabi, M. T. Tabesh, A. Davatgari, M. Miralinaghi, and S. Labi, "Promoting autonomous vehicles using travel demand and lane management strategies," Frontiers in Built Environment, vol. 6, no. 560116, pp. 1-20, 2020.

[26] J. Holguín-Veras and M. Cetin, "Optimal tolls for multi-class traffic: analytical formulations and policy implications," Transportation Research Part A: Policy and Practice, vol. 43, no. 4, pp. 445-467, 2009.

[27] L.-L. Xiao, H.-J. Huang, and R. Liu, "Congestion behavior and tolls in a bottleneck model with stochastic capacity," Transportation Science, vol. 49, no. 1, pp. 46-65, 2015.

[28] Z.-C. Li, W. H. K. Lam, and S. C. Wong, "Step tolling in an activity-based bottleneck model," Transportation Research Part B: Methodological, vol. 101, pp. 306-334, 2017.

[29] M. Miralinaghi, S. Peeta, X. He, and S. V. Ukkusuri, "Managing morning commute congestion with a tradable credit scheme under commuter heterogeneity and market loss aversion behavior," Transportmetrica B: Transport Dynamics, vol. 7, no. 1, pp. 1780-1808, 2019.

[30] J.-P. Wang, H.-J. Huang, and X. Ban, "Optimal capacity allocation for high occupancy vehicle (HOV) lane in morning commute," Physica A: Statistical Mechanics and Its Applications, vol. 524, pp. 354-361, 2019.

[31] X. Yu, V. A. C. van den Berg, and E. T. Verhoef, "Carpooling with heterogeneous users in the bottleneck model," Transportation Research Part B: Methodological, vol. 127, pp. 178200, 2019.

[32] L. Zhong, K. Zhang, Y. Nie, and J. Xu, "Dynamic carpool in morning commute: role of high-occupancy-vehicle (HOV) and high-occupancy-toll (HOT) lanes," Transportation Research Part B: Methodological, vol. 135, pp. 98-119, 2020

[33] F. Mirabel and M. Reymond, "Bottleneck congestion pricing and modal split: redistribution of toll revenue," Transportation Research Part A: Policy and Practice, vol. 45, no. 1, pp. 18-30, 2011. 
[34] L.-J. Tian, H. Yang, and H.-J. Huang, "Tradable credit schemes for managing bottleneck congestion and modal split with heterogeneous users," Transportation Research Part E: Logistics and Transportation Review, vol. 54, pp. 1-13, 2013.

[35] Z.-C. Li and L. Zhang, "The two-mode problem with bottleneck queuing and transit crowding: how should congestion be priced using tolls and fares?" Transportation Research Part B: Methodological, vol. 138, pp. 46-76, 2020.

[36] Z. Lin and H. Yang, "Modal split based on bottleneck model of public transport," China Civil Engineering Journal, vol. 36, no. 7, pp. 1-6, 2003.

[37] R. Arnott, A. de Palma, and R. Lindsey, "Economics of a bottleneck," Journal of Urban Economics, vol. 27, no. 1, pp. 111-130, 1990. 